\title{
Viaggio storico e letterario nella comunità dei Tatari polacco-lituani attraverso Baśnie, podania i legendy polskich Tatarów di Selim Mirza Chazbijewicz
}

\author{
Abstract \\ Literary and historic journeys around \\ the Polish-Lithuanian Tatar community \\ Baśnie, podania i legendy polskich Tatarów \\ by Selim Mirza Chazbijewicz
}

The article deals with the historical reconstruction of the Tatar community in the Grand Duchy of Lithuania. Originally, from Central Asia, after the division of the Mongol Empire the Tatars settled to Eastern Europe giving rise to the Golden Horde. The first migrations of Tatars to Poland took place by the will of the ruler Vitautas in the XIV century. Thanks to their different religious beliefs, the Tatars could keep over the centuries their identity. The Polish-Lithuanian Tatar literature includes two genders: the religious literature and the popular literature. The greatest representative of contemporary Polish literature is the writer Selim Mirza Chazbijewicz. In addition to writing poems and books about the history of Tatars, Chazbijewicz has also dealt with popular literature. The book Fairy tales, stories and legends of the polish Tatars is a collection of 21 stories from the Tatar tradition, passed on orally from generation to generation for centuries. The work is a perfect combination of Judeo-Christian, Islamic and TurkishMongolian traditions.

Key words: Tatars, minorities in Poland, islam in Poland, Selim Mirza Chazbijewicz, Tatar popular literature

Parole chiave: Tatari, minoranze in Polonia, islam in Polonia, Selim Mirza Chazbijewicz, letteratura popolare tatara 
Quando si parla di Polonia in Italia e altri Paesi europei di solito si pensa ad una terra caratterizzata da omogeneità linguistica, etnica, religiosa e culturale. Non bisogna dimenticare, però, che il Paese, in parte favorito dalla sua posizione geografica, nel corso dei secoli è stato un crocevia di culture diverse. Una delle minoranze etnico-religiose presenti da secoli sul territorio polacco e che ha dato un grande contributo militare, economico e culturale alla Polonia è senza ombra di dubbio la comunità tatara. Basti pensare che il grande scrittore e premio Nobel per la letteratura Henryk Sienkiewicz aveva origini tatare.

Attraverso un viaggio storico-letterario scopriremo credenze, usi e costumi di questa minoranza ancora poco conosciuta e sempre più minacciata dal processo di assimilazione. Nel Medioevo, in Occidente, i Tatari erano considerati terribili «guerrieri dell'inferno", giunti dall'Asia centrale e pronti a mettere a ferro e fuoco le città conquistate. A lungo i Tatari e i Mongoli sono stati considerati un unico gruppo etnico. Ma chi erano realmente i Tatari? Da dove venivano? Cosa li spinse a emigrare nell'Europa orientale? E soprattutto erano Turchi o Mongoli? Per rispondere a queste domande occorre fare un salto spazio-temporale per conoscere le origini di questo popolo. II nostro viaggio ha inizio tra le steppe dell'Europa orientale, tra culture apparentemente distanti dal mondo balto-slavo.

I Tatari erano nomadi di lingua turca che vivevano nelle steppe della Mongolia settentrionale, nei territori compresi tra il monte Burhan Haldun e il fiume Herlen. Abili guerrieri, i Tatari erano temuti dai vicini cinesi che, attraverso la Grande Muraglia, avevano creato una barriera difensiva contro di loro e i popoli limitrofi (Grousset 1996: 186-189). I Tatari erano divisi in tribù, ricordiamo alcune delle più note: gli «Otuz Tatar» ovvero i «Trenta Tatari», i «Tokuz Tatar» ovvero i «Nove Tatari» (Vásáry 2005: 9). Nelle fonti cinesi vengono menzionati i «Tatari Neri» e i «Tatari Bianchi» (Eberhard 1947: 259). Sull'origine dell'etnonimo Tatari sono state formulate diverse ipotesi: alcuni studiosi ritengono che il termine derivi dal cinese Ta-ta ovvero 'sporchi, barbari'; secondo l'ipotesi formulata dal filologo Mahmut Kasgarli la parola Tatari deriva dalla radice Tat e significa 'non musulmani', secondo altri storici, invece, Tat-ar significa 'straniero' (Kamalov 2007: 19-20).

Nel Medioevo il termine Tatari è entrato in Occidente attraverso la mediazione dell'arabo e dell'armeno e, come spesso accadeva all'epoca i popoli non cristiani erano tutti etichettati come barbari, così i Tatari e i Mongoli furono considerati un unico popolo. I Tatari, in realtà, erano spesso in conflitto con i Mongoli.

Il clan di Gengis Khan aveva più volte combattuto con questi abili e temibili guerrieri (Yasushi 2008: 1). Yesugei, padre di colui che in seguito sarebbe passato alla storia come Gengis Khan, decise di chiamare il figlio Temujin in onore del valoroso capo tribù tataro da lui sconfitto (Gezici 2013: 11-13). La Mongolia a causa delle continue lotte tra le diverse tribù era facile preda da parte dei popoli limitrofi e per contrastare le invasioni era, dunque, necessario unificare il territorio. Yesugei 
tentò nell'impresa, ma morì prematuramente avvelenato dai Tatari (Cope 2014: 26). Fu suo figlio, il giovane Temujin, che riuscì a porre le fondamenta per l'Impero Mongolo: sottomise dapprima i turchi merkiti del lago Baikal, poi sconfisse i Tatari, i Kerait e in seguito conquistò i territori del Naiman unificando così le diverse tribù della Mongolia (Burgan 2005: 22-28).

Temujin assunse l'appellativo di «Gengis Khan», ovvero «Signore degli Oceani» e divenne il capo supremo dei Mongoli. Unificata la Mongolia, il Gran Khan con uno straordinario esercito di abili arcieri a cavallo partì alla conquista della Cina settentrionale. Dopo la presa di Pechino Gengis Khan si diresse verso Occidente, conquistando in breve periodo il regno musulmano del Khawarism, che comprendeva Persia, Afghanistan e Transoxiana. Da qui l'esercito mongolo si spinse fino alla Russia sud-orientale e poi nuovamente in Cina, ma l'avanzata mongola dovette arrestarsi nel 1227 in seguito alla morte di Gengis Khan. II Signore degli Oceani aveva creato il più vasto impero della storia (Behnke 2008: 40-57).

Dopo la morte di Gengis Khan l'esercito di "diavoli a cavallo» si rimise in marcia, diventando sinonimo di barbarie e terrore in Occidente. In Europa i Mongoli erano confusi con i Tatari - l'esercito mongolo era composto anche da Tatari - e, per la loro ferocia in guerra i «cavalieri del male» furono, per un gioco di parole, chiamati "Tartari», ovvero "creature dell'Inferno». Furono proprio le loro eccezionali doti militari a portarli in quello che ai tempi si chiamava Granducato di Lituania. Prima di arrivare a questo punto, però, bisogna ritornare al 1227, anno della morte di Gengis Khan. Dopo la scomparsa del Signore degli Oceani i territori dell'Impero mongolo furono spartiti tra i quattro figli. Essendo morto Djuci, figlio maggiore di Gengis Khan, la Siberia occidentale passò sotto il controllo del nipote Batu che, approfittando delle rivalità tra i principi russi, riuscì a far capitolare la Rus di Kiev (Marshall 1993: 147-149). Batu proseguì invadendo Polonia, Romania, Boemia ed Ungheria per poi marciare verso Vienna. Non appena Batu ricevette la notizia della morte del Khan Ogedei dovette fare marcia indietro. Al ritorno dalla Mongolia Batu decise di unificare Orda blu (i territori compresi tra il fiume Ural e i monti Carpazi) e Orda bianca (i territori che comprendevano Asia centrale e Siberia meridionale) dando origine al khanato dell'Orda d'oro Batu scelse come capitale la città di Saraj, vicino all'odierna città di Volograd, importante rotta commerciale e centro artigianale (Halperin 1987: 25-28). I Tatari avevano avuto un ruolo fondamentale nella conquista della Rus di Kiev e ben presto la componente turco-tatara prevalse su quella mongola. Non a caso il periodo dell'Orda d'oro è noto come giogo tataro. Di particolare importanza politica e religiosa fu l'islamizzazione del khanato. Il primo khan a convertirsi all'islam fu Berke, fratello di Batu. Berke abbracciò la fede islamica durante un viaggio a Bukhara, in Uzbekistan. Fu con Uzbek Khan (1282-1341) che l'islam diventò la religione ufficiale e in quegli anni l'Orda raggiunse il suo apice. La conversione all'islam non fu 
favorita solo dai floridi rapporti commerciali e dalle relazioni diplomatiche con i Paesi musulmani, ma anche dalla massiccia presenza di musulmani nei territori del khanato (DeWeese 1994: 70-83).

Ben presto però la stabilità dell'Orda cominciò a vacillare in seguito alle guerra civili scoppiate dopo l'uccisione nel 1357 di Gemi Bek, figlio di Uzbek Khan. Approfittando delle debolezze interne dell'Orda il principe di Mosca cercò di liberarsi del giogo tataro, ma il tentativo fallì e per risposta Mosca fu saccheggiata nel 1382 (Crummey 1987: 53). Solo un secolo dopo, nel 1480, quando ormai il khanato, smembrato in tanti piccoli stati e ridotto allo stremo per le continue lotte, Mosca riuscì finalmente a liberarsi del giogo tataro. Nel 1502 la città di Sarai, capitale dell'Orda, fu assediata e poi distrutta dal sovrano del khanato di Crimea Mengli Girai. Il khanato dell'Orda, dopo oltre due secoli, era giunto alla fine.

\section{Tatari del Granducato di Lituania}

La Polonia ebbe un primo contatto con i Tatari nel 1241, quando il Paese, diviso in piccoli principati feudali, fu invaso da un esercito comandato da Batu Khan, nipote di Gengis Khan (Arık 2010: 159). Alcuni Tatari si erano stabiliti nei territori del Granducato di Lituania già a partire dal 1320. Nel 1395 il khan dell'Orda Tokhtamish, dopo essere stato deposto da Tamerlano, trovò rifugio nel Granducato di Lituania, appoggiato dal sovrano Vitoldo (Forbes Manz 1989: 72). Secondo gli Annales di Jan Długosz la data ufficiale in cui i Tatari si stabilirono in Polonia è da considerarsi il 1397 (Mickūnaitè 2006: 188-190).

Tra i primi Tatari del Granducato di Lituania, chiamati in turco Lipka Tatarlar - probabilmente Lipka era il nome della Lituania in tataro - vi erano mercenari, prigionieri di guerra e persone che avevano spontaneamente lasciato i territori dell'Orda a causa delle continue guerre civili. Si stima che le prime comunità tatare non superassero 4000 persone (Arık 2008: 156-161). II Granduca Vitoldo fece stabilire nella città di Trakai un consistente numero di Tatari in cambio di prestazioni militari. Ai nuovi abitanti furono donate terre, garantite libertà di culto e fu concessa loro la costruzione di moschee. I Tatari erano abili guerrieri e spesso ricoprivano le più alte cariche dell'esercito tanto da avere una propria unità militare. I Tatari inoltre erano spesso impiegati come traduttori e ambasciatori tra Polonia e Impero Ottomano. Oltre alla carriera militare e alla diplomazia i Tatari trovavano impiego come artigiani, giardinieri e al servizio dei nobili. I Tatari ebbero anche un ruolo centrale nel commercio con i Paesi dell'Impero Ottomano, tra cui il khanato di Crimea. 
Il clima di tolleranza di cui questa minoranza islamica aveva goduto sin dai primi insediamenti fu turbato nella seconda metà del XVII secolo, quando furono promulgate leggi che limitavano le libertà religiose e i privilegi. A pagare le spese di queste leggi furono anche le donne tatare, accusate di pratiche occulte. Inoltre alla popolazione maschile fu proibito di contrarre matrimonio con donne cristiane. Negli stessi anni il Granducato, a causa delle numerose guerre, stava attraversando una crisi economica che portò a non retribuire i soldati per tre anni. Il clima di intolleranza e il malcontento economico ebbero come conseguenza la migrazione di circa 20000 Tatari nei territori dell'Impero Ottomano. Alcuni tra i Tatari rimasti nel Granducato insorsero nella "Bunt Lipków" ovvero la «rivolta dei Tatari».

Nel 1672 l'esercito turco espugnò la fortezza di Kamieniec Podolski in Ucraina. Ebbe cosi inizio la guerra polacco-ottomana. II conflitto si concluse nel 1676 con la stipulazione del trattato di Zurawno, con il quale la Sublime Porta assicurava alla Polonia un terzo dei territori dell'Ucraina (Rąkowski 2005: 201). Ai Tatari che ritornavano nei territori del Granducato fu concessa l'amnistia e la libertà di culto (Ágoston, Masters 2009: 465). I Tatari, fedeli al Granducato, hanno sempre combattuto in prima linea per difendere la patria, affrontando anche eserciti musulmani. Alla fine del XVIII secolo la Confederazione polacco-lituana ebbe fine e i territori furono spartiti tra Austria, Prussia e Russia. I territori abitati dai Tatari finirono sotto il controllo di Mosca. Tra il XIX e il XX secolo i Tatari svilupparono un vivo interesse verso gli altri paesi musulmani e verso le proprie origini e traduzioni. Nacquero così periodici dedicati alla cultura e storia dei Tatari polacco-lituani e dell'Europa orientale. Attualmente i Tatari sono concentrati prevalentemente nelle città di Danzica, Białystok e nella provincia di Varsavia. Secondo l'ultimo censimento in Polonia il numero dei Tatari ammonterebbe a circa 4000.

\section{La letteratura dei Tatari polacco-lituani}

La letteratura dei Tatari polacco-lituani è unica nel suo genere sia per i temi trattati sia per le peculiarità linguistiche che la rendono una sorta di ponte tra Oriente e Occidente. I Tatari dell'Orda parlavano una lingua appartenente al ceppo turco e che continuarono a parlare quando si stabilirono nel Granducato di Lituania. Con il passare del tempo, però, in parte per i matrimoni con donne del posto, in parte perché tra le varie comunità non vi era omogeneità linguistica, i Tatari cominciarono pian piano a parlare le lingue delle culture ospitanti (Zayonckovski 1939: 339). Questo processo di assimilazione portò alla fioritura

211

Fabrica Litterarum Polono-Italica | 2019, nr 1 (1) 
di una letteratura in cui tradizione orientale e occidentale si fondevano. I Tatari scrivevano in polacco o bielorusso usando i caratteri arabi e slavizzando termini di origine turca, araba, uigur e persiana (Akiner 2009: 69-72). Se dal punto di vista linguistico i Tatari si assimilarono alle popolazioni locali, dal punto di vista religioso un numero consistente continuò a seguire l'Islam, mantenendo vive le proprie tradizioni.

La letteratura dei Tatari polacco-lituani è divisa in due generi: la letteratura popolare e la letteratura religiosa. In questo articolo ci occuperemo principalmente della letteratura popolare. Prima, però, occorre fare una panoramica sulla letteratura religiosa. Si tratta principalmente di traduzioni e commenti del Corano, biografie dei profeti, libri di preghiere e invocazioni. In questa categoria rientrano: kitab, tafsir, khamail e tajwid.

I kitab erano dei libri religiosi scritti a mano da autori anonimi, contenenti precetti religiosi, versetti coranici, storie dei profeti e preghiere. I kitab erano scritti in polacco o bielorusso utilizzando i caratteri arabi (Radziszewska, 2009: 261-263).

Con il termine tafsir in arabo si intende l'interpretazione del Corano. Per i Tatari, invece, i tafsir erano le traduzioni in polacco del testo sacro dell'Islam. Molto probabilmente tale equivoco si deve al fatto che la parola tłumaczenie in polacco significa sia traduzione sia interpretazione. I primi tafsir furono scritti tra le fine del XVI e I'inizio del XVII secolo.

I khamail erano dei libri contenenti scongiuri, preghiere per le varie occasioni e festività, l'oroscopo e l'interpretazione dei sogni.

Infine il tajwid, per gli arabi l'arte della recitazione del Corano. I Tatari con tale termine indicavano i libri che insegnavano a leggere e pronunciare correttamente le sure del Corano.

Per quanto riguarda la letteratura contemporanea ricordiamo il libro pubblicato nel 2010 Versi tatari, la mia eredità, una raccolta di oltre cento poesie scritte da autori diversi, in cui il tema ricorrente è l'identità tatara.

Senza ombra di dubbio il massimo esponente della letteratura tatara contemporanea è Selim Chazbjiewicz. Di origini tatare, nacque a Danzica nel 1955. Dopo essersi laureato in lingua e letteratura polacca all'Università di Danzica, ha conseguito un dottorato in Lettere e Filosofia presso I'Università "Adam Mickiewicz" di Poznań. Dal 1986 al 1991 è stato redattore del periodico quadrimestrale "Życie muzułmańskie" ("Vita musulmana») e dal 1994 al 2003 è stato redattore del periodico "Rocznik Tatarów polskich” («Annuario dei Tatari polacchi»). Nel 2002 ha conseguito il titolo di dottore in Scienze politiche conducendo un lavoro di ricerca sui Tatari di Crimea. Chazbjiewicz ha fondato con Maciej Konopacki e Stefan Mucharski L'Unione dei Tatari della Repubblica Polacca di cui è stato presidente dal 1999 al 2007. Lo scrittore è stato anche imam della moschea di Danzica e dal 1998

212

Fabrica Litterarum Polono-Italica | 2019, nr 1 (1) 
al 2008 co-presidente del Consiglio dei Cattolici e Musulmani Polacchi. La famiglia dello scrittore era originaria di Vilnius dove il suo bisnonno, Ibrahim Smajkiewicz, era imam. La consapevolezza di appartenere alla minoranza tatara ha fortemente influenzato la sua produzione poetico-autobiografica.

L'autore cerca di creare una letteratura tataro-polacca in cui fonde tradizione orientale e occidentale. Chazbijewicz nelle sue poesie riunisce sufismo, esistenzialismo, gnosi e psicoanalisi di Jung. Il poeta cerca il suo «io» nei sogni, nei ricordi e nelle memorie (Czerwiński 2011: 7-8). Le sue prime poesie sono a carattere autobiografico, rievocazioni della sua infanzia e delle tradizioni tatare tramandate dalla famiglia. Altri temi ricorrenti nella poesia di Chazbijewicz sono le steppe lontane, l'eredità spirituale lasciata da Gengis Khan, la spiritualità sufi e il costante richiamo ai riti sciamanici. Chazbijewicz non è solo un poeta, ma è anche autore di diversi saggi sulla storia e letteratura dei Tatari polacco-lituani e di Crimea.

Nel 2012 pubblica il libro Baśnie, podania i legendy polskich Tatarów (Fiabe, racconti e leggende dei Tatari polacchi), una raccolta di 21 racconti e leggende tramandati oralmente per secoli. L'autore è abile nel fondere elementi della tradizione turco-mongolica con elementi della tradizione balto-slava. Inoltre i racconti sono accompagnati da simpatiche illustrazioni realizzate dallo stesso autore. Il libro, gentilmente inviatomi da Chazbijewicz, è stato oggetto di analisi e traduzione per la mia tesi di laurea. Il libro è caratterizzato da un linguaggio scorrevole e lineare e presenta termini di origine araba, turca e farsi. I racconti sono caratterizzati generalmente da periodi brevi e da abbondanti descrizioni. La particolarità del libro è l'influenza orientale non solo dal punto di vista linguistico, ma anche stilistico.

Chazbijewicz, come spiega nella prefazione, decide di dare ai racconti la forma della maqama, un genere di prosa rimata che fiorì nel mondo arabo tra IX e XII secolo e che si diffuse anche in Andalusia (Meisami, Starkey 1998: 507-508). Come è tipico della tradizione orientale all'inizio di ogni racconto il narratore riferisce di aver sentito la storia da qualcun altro, in questo caso Selim Mirza, un alter ego dell'autore. L'ultimo racconto si apre con una lunga catena di trasmettitori che inizia con Selim Mirza e si conclude con Ignacy Chodźko, poeta polacco-lituano vissuto nell'Ottocento. Lo scopo della catena dei trasmettitori è quello di dare veridicità ai racconti e di viaggiare a ritroso nel tempo. Nel libro ci sono numerosi riferimenti geografici sia a città dell'Europa orientale sia a città dell'Asia centrale e del mondo islamico.

Per quanto riguarda i nomi dei personaggi dei racconti Chazbijewicz utilizza sia nomi tipicamente slavi sia nomi di origine araba e turco-mongolica. Inoltre alcuni dei protagonisti dei racconti sono personaggi storici. La presenza di figure realmente esistite è importante per l'inquadramento storico del racconto. Spesso i personaggi storici fungono da sfondo, hanno un ruolo marginale, mentre il ruolo

213

Fabrica Litterarum Polono-Italica | 2019, nr 1 (1) 
da protagonista è affidato a personaggi di pura fantasia. Tra i personaggi storici polacco-lituani e del mondo slavo in genere, nelle storie compaiono nomi come quello di Krzysztof Radziwiłł, Stefano I di Polonia e Ivan il Terribile. Tra i personaggi storici dell'Asia centrale compaiono sullo sfondo dei racconti Tamerlano e Gengis Khan. Oltre alle figure storiche e di pura fantasia alcuni racconti hanno per protagonisti i profeti del Cristianesimo e dell'Islam. Spesso nei racconti dei profeti l'autore attinge sia a fonti giudaico-cristiane sia a fonti islamiche.

Un topos letterario dei racconti dei Tatari polacco-lituani è quello del viaggio verso Oriente. Altra figura spesso ricorrente nel libro è quella dell'oste, spesso connotata negativamente come nei racconti Abid e Kotun di Baghdad. Altra peculiarità del libro di Chazbijewicz è l'abbondante uso di termini di origine araba e turca. Il titolo originale del secondo racconto è Podanie o fałdzeju che ho tradotto in italiano con Racconto del mago. Il termine fałdzej è un calco del termine turco falci, letteralmente significa 'cartomante', 'indovino'. Falci è composto dal termine fal, che significa 'sorte', 'destino', e dal suffisso ci che in turco si usa per indicare la professione.

Un altro termine di derivazione turca presente nel libro è kurhan, in italiano tradotto con kurgan, ovvero i tumuli funerari sacri delle popolazioni turco-altaiche di forma conica o semi-circolare. Chazbijewicz, inoltre fa abbondante uso di terminologia islamica. Uno dei racconti ha per protagonista un «mulla», letteralmente 'signore, padrone' o 'maestro'. Presso le popolazioni tatare il termine è entrato dal turco molla e indica il clero islamico. In un altro racconto il protagonista vede in sogno un "derviscio». La parola deriva dal farsi e significa 'povero', 'indigente'. Con tale appellativo si chiamavano i sufi, asceti islamici che conducevano una vita in mistica povertà.

Oltre alla terminologia religiosa e ai calchi e prestiti linguistici presi dall'arabo, dal turco e dal persiano, nel libro l'autore ricorre a termini politico-militari di origine slava e germanica. Nel racconto Storia del mago Chazbijewicz usa il termine kniaź, in italiano tradotto come duca o principe, termine che nelle lingue slave indica una persona di ceto nobile. Un altro termine che ricorre spesso nei racconti è hetman, in italiano tradotto come etmano, titolo con il quale nell'esercito del Granducato di Lituania veniva designato il grado più elevato e in seguito il capo politico (Lerski 1999: 196). Ogni racconto è ricco di riferimenti storici, religiosi e culturali.

Passiamo ora a un'analisi dettagliata di alcuni dei racconti. Il primo personaggio a comparire sulla scena, e che sarà una costante in tutti i racconti, è Selim Mirza, un alter ego dell'autore. Selim Mirza (1877) è un romanzo dello scrittore polacco di origini tatare Henryk Sienkiewicz. Mirza è un titolo nobiliare di origine persiana che deriva dal termine Amirzadeh letteralmente 'figlio del principe, di sangue reale' (Bonnefoy 1933: 331).

214

Fabrica Litterarum Polono-Italica | 2019, nr 1 (1) 
Il primo racconto del libro che ho tradotto come Storia del lupo coraggioso, ha per protagonista un lupo, animale presente in molte leggende e storie di diverse culture in varie parti del mondo. II racconto è un tipico esempio di fusione tra cultura turco-mongolica e cultura balto-slava. La storia all'inizio è ambientata nel monte Burhan Haldun, in Mongolia, monte considerato sacro da Gengis Khan. II racconto inizia in un'epoca remota, ai tempi di Tengri Khan, il "Signore degli spiriti», il "Dio dei cieli». Tengri era il Dio supremo al quale i Mongoli, compreso Gengis Khan, erano soliti rivolgere le preghiere e chiedere protezione prima di una guerra. Il lupo vive tra i monti Altai, seminando terrore tra $\mathrm{i}$ cacciatori. Divenuto una leggenda, lentamente scompare dalla scena. Improvvisamente Chazbijewicz con un salto spazio-temporale passa dall'ambientazione in Asia centrale all'ambientazione nel mondo balto-slavo. II lupo infatti ricompare ed è protagonista del sogno di Gediminas (1275-1341), il primo Granduca di Lituania nonché fondatore della città di Vilnius nel 1323. Nell'ultima parte del racconto è chiaro il riferimento alla leggenda del lupo di ferro, che presenta analogie con la lupa capitolina.

Narra la leggenda che il Granduca Gediminas stava cacciando in una foresta sacra. Stancatosi si addormentò nel bosco. In sogno gli apparve un enorme lupo di ferro che dalla sommità di un colle ululava molto forte. Al risveglio il duca chiese a un sacerdote di interpretare il sogno. Questi gli rispose che il lupo era stato mandato in sogno dagli dei perché il duca fondasse una città. Nacque così la città di Vilnius (Suziedelis 1997: 136). Il lupo è, dunque, una figura importante sia nella mitologia turco-mongolica sia nella mitologia balto-slava.

Nella prima parte del racconto il lupo rievoca il leggendario Bozkurt, il lupo celeste della mitologia turco-mongolica, animale sacro inviato da Tengri Khan e da cui sarebbe disceso Gengis Khan e il popolo turco (Sapolyo 1967: 54, 137). L'autore fonde i due miti per creare senso di continuità tra i popoli turco-mongolici e i balto-slavi.

Il secondo racconto, Storia del mago, ha per protagonista una bellissima ragazza di nome Leila, innamorata perdutamente di un giovane principe discendente della famiglia Emir Valil-Lowczyk, una delle più importanti famiglie dell'aristocrazia tatara. A causa del diverso ceto sociale la ragazza non è corrisposta. Un giorno Leila si imbatte in un mago che le promette di aiutarla a conquistare il suo amato attraverso un rituale magico. Condizione necessaria per la realizzazione dell'incantesimo è mantenere il silenzio. Proprio quando il principe stava per cedere, la ragazza sbadatamente si lascia sfuggire il nome del principe davanti a una sua amica. L'incantesimo, così, è annullato.

La giovane fanciulla si chiama Leila, nome di origine araba che letteralmente significa 'notte, bella come la notte'. II nome ebbe vasta popolarità nel mondo musulmano, soprattutto nel folklore arabo-persiano e turco grazie al celebre poema

215

Fabrica Litterarum Polono-Italica | 2019, nr 1 (1) 
Leila e majnun. II poema narra l'impossibile storia d'amore tra Qays e la bellissima Leila a causa della disapprovazione del padre della ragazza. Qays impazzisce per l'impossibilità di coronare il suo sogno d'amore e per questo viene chiamato majnun letteralmente 'posseduto dai Jin, pazzo'.

La protagonista del racconto di Chazbijewicz vive un amore infelice che la tormenta portandola quasi alla pazzia, per questo, richiamandosi alla tradizione arabo-persiana, la ragazza viene chiamata Leila.

Un altro racconto interessante è II paggio tataro. II racconto è ispirato a una leggenda tratta da una storia vera, molto popolare in Polonia. Protagonisti della storia sono Janusz Radziwiłł (1579-1620), sua moglie Sophia Oledkovich Radziwiłł (1585-1612) e il giovane tataro Smolski, al servizio del principe. Si racconta che la principessa e il paggio si innamorarono perdutamente. II principe li colse in flagrante e condannò a morte il giovane tataro. II paggio, trovato in possesso dei gioielli e delle monete che gli aveva donato la principessa, fu accusato di furto e ucciso per annegamento.

Il principe Radziwiłł nel racconto è definito traditore perché aveva tradito la patria nel "Potop szwedzki" ovvero il "Diluvio svedese», termine con cui si intende I'invasione che subì la Confederazione polacco-lituana da parte dell'Impero svedese tra il 1655 e il 1660. Poiché Radziwiłł aveva stipulato accordi con la Svezia (Gerasimova 2009: 447-463) nella cultura popolare polacca il principe è descritto come un personaggio negativo. La protagonista femminile del racconto è Santa Sophia di Slustk (1585-1612), molto venerata tra gli ortodossi. Rimasta orfana in giovane età, fu data in moglie a soli quindici anni al principe Janusz Radziwiłł. Sophia continuò a seguire il credo ortodosso anche dopo le nozze e la santa è considerata dai fedeli simbolo di devozione e generosità.

I Tatari, come già detto, erano musulmani e per questo spesso nei loro racconti e leggende si parla dei precetti dell'Islam. Uno dei racconti che ha per protagonista un giovane musulmano è Abid. II ragazzo un giorno si reca in un osteria dove a servirlo trova una donna. La locandiera costringe il giovane a bere I'alcol. Ubriacatosi Abid abusa della donna e in seguito uccide il figlio dell'oste. II racconto spiega dunque il motivo per cui un buon musulmano dovrebbe astenersi dall'alcol. La storia di Abid e degli effetti negativi dell'alcol ci riporta a una leggenda sul Profeta Muhammad diffusa in Occidente nel Medioevo. Secondo la leggenda, il Profeta dell'Islam, dopo essersi ubriacato, uccise il monaco Buhira. Resosi conto delle gravi conseguenze a cui poteva portare l'alcol, il Profeta proibì alla comunità musulmana l'uso di tali bevande.

Concludiamo con un racconto che ha per protagonista il Profeta Abramo. La storia è di particolare interesse per i riferimenti storico-religiosi che attingono a fonti cristiane e musulmane. All'inizio del racconto l'autore menziona il re Nimrod. II Profeta Abramo nacque infatti durante il suo regno ed è considerato il primo

216

Fabrica Litterarum Polono-Italica | 2019, nr 1 (1) 
patriarca per Ebrei, Cristiani e Musulmani, nonché il primo monoteista. Secondo il Corano «Abramo non era né giudeo, né nazareno, ma puro credente e musulmano. E non era uno degli associatori» (Corano 3:67). Abramo è considerato dai musulmani il primo costruttore della kaaba alla Mecca. Figlio di uno scultore di idoli, da bambino fu nascosto in una grotta che si trova a Sanliurfa, a Sud-est della Turchia, per scampare al massacro di bambini ordinato dal re Nimrod.

Nel racconto l'autore ripercorre la vita del Profeta dalla sua nascita fino alla descrizione del miracolo grazie al quale Abramo, condannato a morte dal re, riuscì a salvarsi dalle fiamme per volere di Dio. L'episodio a cui si riferisce Chazbijewicz è narrato nel Corano. Abramo fu gettato in una pira per volere del re Nimrod, ma fu salvato da Dio che, facendo comparire una sorgente d'acqua, spense le fiamme. Si conclude qui la prima parte del nostro viaggio verso le leggende e i racconti dei Tatari polacco-lituani.

\section{Bibliografia}

Ágoston Gábor, MASTERS Bruce (2009): Encyclopedia of the Ottoman Empire. New York.

AKINER Shirin (2009): Religious Language of a Belarusian Tatar Kitab. A Cultural Monument of Islam in Europe. Wiesbaden.

ARIK Sabire (2008): Polonya-Litvanya Tatar Türkleri (Lipkalar). "Modern Türklükar Araştırmaları Dergisi" [Ankara], sayı 5/3, p. 156-161.

ARIK Sabire (2010): Kuruluştan XVII Yüzyıla Polonya Tarihi. Ankara.

BEHNKE Alison (2008): The Conquests of Genghis Khan. Minneapolis.

BoNNEFOY Yves (1933): Asian mythologies. Chicago.

BURGAN Michael (2005): Empire of the Mongols. New York.

CHAZBIJEWICZ Selim (2012): Baśnie, podania i legendy polskich Tatarów. Białystok.

COPE Tim (2014): On the Trail of Genghis Khan: An Epic Journey Through the Land of the Nomads. London.

CRUMMEY Robert (1987): The Formation of Muscovy 1300-1613. New York.

CZERWIŃSKI Grzegorz (2011): Panorama de la littérature polono-tatare auX XXe et XXI siècle. "Slavica bruxellensia" [Bruxelles], N. 7.

DEWeESE Devin (1994): Islamization and Native Religion in the Golden Horde. Philadelphia.

EBERHARD Wolfram (1947): Çin tarihi. Ankara.

FORBES MANZ Beatrice (1989): The Rise and Rule of Tamerlane. Cambridge.

GERASIMOVA Irina (2009): Between Vilna and Konigsberg: a history of one flight, August 1655. "Ajalooline Ajakiri" [Tartu], N. 3-4.

GeZICI Aytekin (2013): Cengiz Han. Ankara.

217

Fabrica Litterarum Polono-Italica | 2019, nr 1 (1) 
GRouSSET Rene (1996): Bozkur imparatorluğu. İstanbul.

HALPERIN Charles J. (1987): Russia and the Golden Horde: The Mongol Impact on Medieval Russian History. Bloomington.

Kamalov Ilyas (2007): Avrasya Fatihi Tatarlar. İstanbul.

LERSKI George J. (1999): Historical Dictionary of Poland, 966-1945. Westport.

MARSHALL Robert (1993): Storm from the East: From Genghis khan to Khubilai khan. Los Angeles.

MeISAmI Julie Scott, StARkey Paul (1998): Encyclopedia of Arabic literature. Vol. 2. London.

MıcKūNAITĖ Giedrè (2006): Making a Great Ruler: Grand Duke Vytautas of Lithuania. Budapest.

RADZISZEWSKA Iwona (2009): Język ksigg religijnych Tatarów litewsko-polskich, funkcjonujq̨cych w środowisku muzułmanów polskich. „Linguistica Copernicana” [Toruń], $\mathrm{nr} 2$ (2).

RĄKOWSKI Grzegorz (2005): Polska egzotyczna. Warszawa.

SAPolyo Enver Behnan (1967): Türk Efsaneleri. Istanbul.

SUZIEDELIS Saulius (1997): Historical Dictionary of Lithuania. Maryland.

VÁSÁRY István (2005): Cumans and Tatars: Oriental Military in the Pre-Ottoman Balkans 1185-1365. Cambridge.

YASUSHI Inoue (2008): The Blue Wolf: a novel of the life of Chinggis Khan. New York. ZAYONCKOVSKI Ananyas (1939): Lehistan arazisinde Türk unsurlari. Türklük. Istanbul.

\section{Abstrakt \\ Literackie wędrówki i historyczne podróże wokół społeczności Tatarów polsko-litewskich Baśnie, podania i legendy polskich Tatarów autorstwa Selima Mirzy Chazbijewicza}

W artykule podjęta została próba kulturowej i historycznej rekonstrukcji społeczności Tatarów zamieszkałych na terytorium Wielkiego Księstwa Litewskiego. Tatarzy, pochodzący z Azji Środkowej, po podziale imperium mongolskiego udali się do Europy Wschodniej, dając początek chanatowi Złotej Ordy. Pierwsze migracje Tatarów na ziemie polskie z woli Wielkiego Księcia Litwy Witolda datuje się na wiek XIV. Dzięki przynależności do religii muzułmańskiej Tatarzy zdołali na przestrzeni wieków ocalić własną tożsamość. Twórczość literacką Tatarów polsko-litewskich można podzielić na dwa typy literatury: religijną i popularną. Najznakomitszym przedstawicielem polskiej współczesnej literatury tatarskiej jest Selim Mirza Chazbijewicz. Twórca, oprócz poezji i eseistyki, interesował się popularną literaturą tatarską. Książka Baśnie, podania i legendy polskich Tatarów to zbiór 21 opowiadań z tradycji tatarskiej, przekazywanych 
przez wieki ustnie. Książka ta jest dowodem na występowanie kulturowego splotu tradycji judeochrześcijańskiej, islamskiej i turecko-mongolskiej.

Słowa kluczowe: Tatarzy, mniejszości w Polsce, islam w Polsce, Selim Mirza Chazbijewicz, popularna literatura tatarska 